\title{
Patches with short boundaries
}

\author{
G. Brinkmann, S. Van den Eynde \\ Applied Mathematics, Computer Science and Statistics, Ghent University, Krijgslaan \\ 281-S9, 9000 Ghent, Belgium
}

\begin{abstract}
In this article we prove bounds for the boundary length of patches with a given set of bounded faces. We assume that with $t$ the number of given triangles, $q$ the number of quadrangles, and $p$ the number of pentagons, the curvature $3 t+2 q+p$ is at most 6 and that at an interior vertex exactly 3 faces meet. There is no restriction on the number of faces with size 6 or larger. We prove that one gets a patch with shortest boundary if one arranges the faces in a spiral order and with increasing size. Furthermore we give explicit formulas that allow to determine all boundary lengths that occur for patches with given numbers $p, q$ and $t<2$ and no bounded face larger than 6 .

The patches studied in this article occur as subgraphs of 3-regular graphs in mathematics as well as models for planar polycyclic hydrocarbons in chemistry where the bounds allow to decide on the (theoretical) existence of molecules for a given chemical formula.
\end{abstract}

Keywords: patch, boundary, spiral

\section{Introduction}

Connected bridgeless plane graphs in which all bounded faces are hexagons, all vertices have maximum degree 3 and all vertices not in the boundary of the outer, unbounded face have degree exactly 3 are especially interesting as they are models for benzenoids and fusenes in chemistry, correspond to closest packings of spheres, and occur as subgraphs of cubic plane graphs. Several papers - the earliest one being [10] - independently determine the shortest possible length of the boundary as a function of the number of

Email addresses: Gunnar.Brinkmann@ugent.be (G. Brinkmann), Simon.VandenEynde@UGent.be (S. Van den Eynde) 
hexagons. The central result of these papers is that you get the shortest possible boundary if you arrange the hexagons in a spiral fashion around a central hexagon. With this key result it is possible to decide on the existence of benzenoids for a given chemical formula and determine sequences of formulas with an equal number of isomers (see e.g. [7]). With the advent of fullerenes (that is: carbon molecules corresponding to 3-regular plane graphs with only pentagons and hexagons) also patches with bounded faces of size 5 (but still maximum face size 6 of a bounded face) became increasingly interesting and in [2] the result from [10] was generalized to patches allowing up to 6 pentagons in an otherwise hexagonal patch. The result from which the formulas for the smallest boundary length as a function of the number $p$ of pentagons and $h$ of hexagons was deduced is that the smallest boundary is obtained by arranging the faces in a spiral fashion starting with the pentagons. This result found applications in mathematics, chemistry, algorithms and the interplay between these disciplines (see e.g. [1], [5], [6] ). Already at that time it was conjectured that by using similar methods as in [2] the result could be further generalized to patches also allowing $t$ triangles and $q$ quadrangles - as long as the curvature $3 t+2 q+p$ is at most 6 and that in this case spirals using the faces in increasing order of their size give shortest boundaries.

In her Diploma thesis [8] Anke Egging attacked this problem and announced that result as well as formulas for the minimal boundary length for given $t, q, p$, and number $h$ of hexagons. The result was applied in the $\mathrm{PhD}$ thesis [11] of Claudia Justus, who noted and corrected an error in the formulas. Although these results were not published in a journal, they had some impact and were used by other researchers (see [9],[13]). In the proof of one of Barnette's conjectures [12], František Kardoš used a version of this theorem with curvature at most 5 and a special structure of the boundary cycle for which he provided his own proof. Later it was detected that in [8] not only the formulas, but also the proof of the central theorem on which all other results are based - the minimality of spirals - contained an error that could not easily be corrected. Egging proved the minimality of spirals among all patches with the same set of bounded faces as a direct consequence of another theorem and not only the proof of the stronger theorem, but also the stronger theorem itself are wrong.

In this article we prove the minimality of spirals in an even more general context also allowing bounded faces of size more than 6 and give the corrected formulas as described in [11] for face size at most 6 . 


\section{Basic definitions and results}

We assume all plane graphs to be embedded in the plane - not the sphere. To this end there is a unique unbounded or outer face.

Some elementary proofs are left to the reader. The basic strategy and some proofs follow the lines of [2].

Definition 1. The face sequence of a plane graph with $l$ bounded faces is the sequence $\left(s_{1}, s_{2}, \ldots, s_{l}\right)$ of face sizes of bounded faces, sorted in increasing order. A sequence $s=\left(s_{1}, \ldots, s_{l}\right)$ for some $l$ is called simple, if the curvature $c(s)=\sum_{s_{i}<6}\left(6-s_{i}\right)$ is at most $6, s_{1} \geq 3$ and the entries are in increasing order.

The excess $\operatorname{ex}(s)$ of a simple sequence $s=\left(s_{1}, \ldots, s_{l}\right)$ is defined as $\operatorname{ex}(s)=$ $\sum_{i=1}^{l}\left(s_{i}-6\right)$.

A c-6-patch (short for curvature-6-patch) is a finite simple plane 2-connected graph with a simple face sequence and maximum degree 3 in which each vertex not in the boundary of the unbounded face has degree exactly 3 . We denote a c-6-patch $P$ with face sequence $s$ by $s$-patch and also define $\operatorname{ex}(P)=\operatorname{ex}(s)$. Note that by definition we have $\operatorname{ex}(P) \geq-6$.

For a simple sequence $s$, an $(s, k)$-patchset $\bar{P}$ is a set of $k$ (nonempty) c-6-patches $P_{1}, \ldots, P_{k}$ so that $s$ is the sequence of all sizes of bounded faces in the patches sorted in increasing order. We assume the patches to be embedded in a way that no patch is embedded in a bounded face of another patch.

The boundary length $b(P)$ of a c-6-patch $P$ is the number of vertices (or equivalently: edges) in the boundary of the unbounded face. The boundary length $b(\bar{P})$ of an $(s, k)$-patchset $\bar{P}=\left\{P_{1}, \ldots, P_{k}\right\}$ is defined as $b(\bar{P})=$ $\sum_{i=1}^{k} b\left(P_{i}\right)$. We denote the number of vertices in the boundary that have degree 2 , resp. 3 , by $v_{2, b}(P)$, resp. $v_{3, b}(P)$. The number of bounded faces that share an edge with the unbounded face is denoted by $f_{b}(P)$.

For a simple sequence $s=\left(s_{1}, \ldots, s_{l}\right)$ and $1 \leq k \leq l$ we define

$\min (s, k)=\min \{b \mid \exists(s, k)$-patchset $\bar{P}$ with $b(\bar{P})=b\}$

$\min (s)=\min \{\min (s, k) \mid 1 \leq k \leq l\}$

$\max (s, k)=\max \{b \mid \exists(s, k)$-patchset $\bar{P}$ with $b(\bar{P})=b\}$

Observing that inner duals (that is: the dual graph without the vertex corresponding to the outer face) of c-6-patches are connected, it is easy to determine $\max (s, k)$ :

Lemma 2. For a simple sequence $s=\left(s_{1}, \ldots, s_{l}\right)$ and an $(s, k)$-patchset $\bar{P}$ we have that $b(\bar{P})=\max (s, k)$ if and only if the inner dual of each patch is 
a tree.

As a consequence we have $\max (s, k)=\sum_{i=1}^{l}\left(s_{i}-2\right)+2 k$.

Proof. Let $e_{2}(\bar{P})$ (resp. $e_{2}(P)$ ) denote the number of edges in $\bar{P}$ (resp. in a c-6-patch $P$ ) that are contained in two bounded faces. In a patch with $f$ bounded faces we have $e_{2}(P) \geq f-1$ and $e_{2}(P)=f-1$ if and only if the inner dual of $P$ is a tree. This implies that $e_{2}(\bar{P}) \geq l-k$. The edges contained in two bounded faces are exactly the edges not in the boundary. Summing up all face sizes of bounded faces and subtracting the interior edges, which are not in the boundary, but were counted twice, we get

$$
b(\bar{P})=\sum_{i=1}^{l} s_{i}-2 e_{2}(\bar{P}) \geq \sum_{i=1}^{l} s_{i}-2(l-k)=\sum_{i=1}^{l}\left(s_{i}-2\right)+2 k
$$

with equality if and only if the inner dual of every patch is a tree.

The fact that such patch sets do in fact exist, is elementary.

Lemma 3. For a c-6-patch $P$ we have

$v_{2, b}-v_{3, b}=6+\operatorname{ex}(P)$ which implies

- $b(P)=2 v_{3, b}+6+\operatorname{ex}(P)$ and

- $v_{2, b} \geq v_{3, b}$

Proof. Let $s=\left(s_{1}, \ldots, s_{l}\right)$ be the face sequence of $P$. Summing up the sizes of all bounded faces, we count all interior vertices three times, boundary vertices with degree 3 two times and boundary vertices with degree 2 once. For the number $v$ of vertices this gives

$$
v=\frac{\sum_{i=1}^{l} s_{i}+v_{3, b}+2 v_{2, b}}{3} .
$$

Summing up all face sizes - including the size of the outer face - we count each edge twice and get for the number $e$ of edges that

$$
e=\frac{\sum_{i=1}^{l} s_{i}+v_{3, b}+v_{2, b}}{2}
$$

Inserting this together with $1+l$ for the number $f$ of all faces into the Euler formula $v-e+f=2$ we get 


$$
\frac{\sum_{i=1}^{l} s_{i}+v_{3, b}+2 v_{2, b}}{3}-\frac{\sum_{i=1}^{l} s_{i}+v_{3, b}+v_{2, b}}{2}+l+1=2 .
$$

Simplifying that gives

$$
\underbrace{-\left(\sum_{i=1}^{l} s_{i}-6 l\right)}_{-\operatorname{ex}(P)}-v_{3, b}+v_{2, b}=6 .
$$

This implies the formulas in the lemma.

Lemma 4. For a simple sequence $s=\left(s_{1}, \ldots, s_{l}\right)$ and $1 \leq k<l$ we have $\min (s, k)<\min (s, k+1)$, so especially $\min (s)=\min (s, 1)$.

Proof. We call a boundary edge with both endpoints of degree 2 a convex edge.

Let $\left\{P_{1}, \ldots, P_{k+1}\right\}$ be a patchset realizing $\min (s, k+1)$. If there are two patches $P_{i}, P_{j}$ each with a convex edge, we can identify these edges and get an $(s, k)$ patchset with boundary length $\min (s, k+1)-2$.

By Lemma 3 each patch $P_{i}$ with $\operatorname{ex}\left(P_{i}\right)>-6$ has a convex edge, so the only case where it is possible that no two such patches exist, is $k+1=2$ and one patch - w.l.o.g. $P_{1}-$ has $\operatorname{ex}\left(P_{1}\right)=-6$ and the degrees of the vertices in the boundary alternate between 2 and 3 and the other patch $P_{2}$ contains only faces of size at least 6 and therefore satisfies $\operatorname{ex}\left(P_{2}\right) \geq 0$.

If $P_{2}$ has three neighbouring vertices of degree 2 in the boundary, the path connecting these vertices can be identified with a path of length two with a central vertex of degree 3 in the boundary of $P_{1}$ to get a patch with a shorter boundary.

If there are at most two neighbouring vertices with degree 2 in the boundary, there is a hexagon in the boundary of $P_{2}$ containing a convex edge: if all bounded faces in the boundary with convex edges had size at least 7 , we could remove one of the vertices of each convex edge and get a patch $P^{\prime}$ without convex edges but $\operatorname{ex}\left(P^{\prime}\right) \geq 0$ - contradicting Lemma 3. Note that under these circumstances a face with $m$ convex edges has size at least $4 m$ (three boundary edges and one internal edge for each convex edge), so that we can remove a vertex of degree 2 of each convex edge and the remaining face size is still at least 6 . So let $h$ be a hexagon with a convex edge. This must be the only convex edge of $h$. Removing $h$, the result is either connected or falls into two parts - each with a convex edge it shared with $h$. 
After possibly identifying two parts along these convex edges we get a patch $P_{-}$with boundary length at most $b\left(P_{2}\right)$.

The boundary of $P_{2}$ contains at least 6 vertices of degree 3 neighbouring only vertices of degree 2 (a consequence of Lemma 3), so there is a vertex $v$ not neighbouring a vertex of $h$ that has this property also in $P_{-}$. We can identify opposite vertices of $h$ once with $v$ and once with a vertex of degree 3 in $P_{1}$ and get a patch with boundary length $b\left(P_{1}\right)+b\left(P_{-}\right)-4+2 \leq$ $b\left(P_{1}\right)+b\left(P_{2}\right)-2$.

Lemma 5. For every c-6-patch $P$ with at least two bounded faces we have $f_{b}(P) \leq v_{3, b}(P)$ and $f_{b}(P)=v_{3, b}(P)$ if and only if the intersection of each boundary face with the boundary is connected.

Proof. The proof is identical to that of Remark 3 in [2], which states the same result for a more restricted class.

We can fix a direction to traverse the boundary and map each boundary vertex with degree 3 to the boundary face containing the edge following the vertex in the chosen direction. This mapping is always surjective (so $\left.f_{b}(P) \leq v_{3, b}(P)\right)$ and bijective if and only if the intersection of each boundary face with the boundary is connected, so that $f_{b}(P)$ is equal to $v_{3, b}(P)$ exactly in this case.

Lemma 6. Let $P$ be a c-6-patch. Then $b(P)-\operatorname{ex}(P)$ is even.

Proof. If $C$ is the set of edges of $P$ that is contained in two bounded faces and $s=\left(s_{1}, \ldots, s_{l}\right)$ is the face sequence, then

$$
b(P)=\sum_{i=1}^{l} s_{i}-2|C|
$$

and

$$
b(P)-\operatorname{ex}(P)=\sum_{i=1}^{l} s_{i}-2|C|-\sum_{i=1}^{l}\left(s_{i}-6\right)=6 l-2|C| .
$$

Lemma 7. Let $P$ be a $c$-6-patch with at least one interior face and let $P_{-}$ be the patchset obtained by removing all boundary faces of $P$. Then

$$
b\left(P_{-}\right)-\operatorname{ex}\left(P_{-}\right) \leq b(P)-\operatorname{ex}(P)-2\left(6+\operatorname{ex}\left(P_{-}\right)\right)
$$

and we have equality if and only if each face in the boundary of $P$ has exactly two boundary faces as neighbours. 
Proof. The inner dual induced by the boundary faces of $P$ is connected and due to the fact that $P$ has an interior face, it has at least one cycle. So it has at least $f_{b}(P)$ edges, or in other words: the boundary faces share at least $f_{b}(P)$ edges.

The boundary edges of $P_{-}$are exactly those edges that are contained in a boundary face of $P$, but are neither in the boundary of $P$ nor shared by another boundary face, so with $s_{1}^{\prime}, \ldots, s_{f_{b}(P)}^{\prime}$ the sizes of the boundary faces we have

$$
b\left(P_{-}\right) \leq \sum_{i=1}^{f_{b}(P)} s_{i}^{\prime}-b(P)-2 f_{b}(P)
$$

We can write $\sum_{i=1}^{f_{b}(P)} s_{i}^{\prime}$ as

$$
\sum_{i=1}^{f_{b}(P)} s_{i}^{\prime}=\sum_{i=1}^{f_{b}(P)}\left(s_{i}^{\prime}-6\right)+6 f_{b}(P)=\operatorname{ex}(P)-\operatorname{ex}\left(P_{-}\right)+6 f_{b}(P),
$$

and therefore

$b\left(P_{-}\right) \leq \operatorname{ex}(P)-\operatorname{ex}\left(P_{-}\right)+6 f_{b}(P)-b(P)-2 f_{b}(P)=\operatorname{ex}(P)-\operatorname{ex}\left(P_{-}\right)+4 f_{b}(P)-b(P)$.

By Lemma 3 we can replace $b(P)$ and get

$b\left(P_{-}\right) \leq \operatorname{ex}(P)-\operatorname{ex}\left(P_{-}\right)+4 f_{b}(P)-2 v_{3, b}-6-\operatorname{ex}(P)=4 f_{b}(P)-\operatorname{ex}\left(P_{-}\right)-2 v_{3, b}-6$.

By Lemma 5 we can replace $f_{b}(P)$ by $v_{3, b}$ and by Lemma 3

$$
b\left(P_{-}\right) \leq 2 v_{3, b}-6-\operatorname{ex}\left(P_{-}\right)=b(P)-6-\operatorname{ex}(P)-6-\operatorname{ex}\left(P_{-}\right) .
$$

Subtracting ex $\left(P_{-}\right)$on both sides we get the first result in the lemma.

In order to have equality we have to have equality in Lemma 5, which is the case if and only if the intersection of each boundary face with the boundary is connected. If a boundary face $f$ shared only one edge with another boundary face $f^{\prime}$ and there are more than two faces, then at least one of $f, f^{\prime}$ would have a disconnected intersection with the boundary. So each boundary face shares at least 2 edges with other boundary faces, but then our estimation that the boundary faces share at least $f_{b}(P)$ edges is 
sharp if and only if every boundary face shares exactly two edges with other boundary faces. This property alone also implies that the intersection of each boundary face with the boundary is connected, so that all inequalities become equalities.

Definition 8. A spiral patch - or spiral for short - is a c-6-patch with bounded faces $f_{1}, \ldots, f_{l}$ for which a bijective mapping sp $:\{1, \ldots, l\} \rightarrow$ $\left\{f_{1}, \ldots, f_{l}\right\}$ exists so that with $S_{k}$ the subgraph consisting of the vertices and edges of the faces $\operatorname{sp}(1), \ldots, \operatorname{sp}(k)$ we have

- for $1 \leq i \leq l S_{i}$ is a c-6-patch with bounded faces $\operatorname{sp}(1), \ldots, \operatorname{sp}(i)$ and face $\operatorname{sp}(i)$ is in the boundary

- for $1<i \leq l$ face $\operatorname{sp}(i)$ has a connected intersection $C$ with $S_{i-1}, C$ contains an edge of $\operatorname{sp}(i-1)$ and for $i>2$ it also contains an edge of the face $\operatorname{sp}(j)$ with $j$ the smallest index so that $\operatorname{sp}(j)$ is in the boundary of $S_{i-1}$

We call $\operatorname{sp}(\cdot)$ a spiral numbering and for a given simple sequence $s=$ $\left(s_{1}, \ldots, s_{l}\right)$ we call a patch the spiral for $s$ (and denote it by $S_{s}$, resp. $S_{s, k}$ for $k \leq l$ if restricted to $\left.s=\left(s_{1}, \ldots, s_{k}\right)\right)$ if it has a spiral numbering so that for $1 \leq i \leq l$ we have that $\operatorname{sp}(i)$ has size $s_{i}$.

Spirals do not exist for every increasing sequence - e.g. not for $(3,4,5,5,6,6,6)$ - but they do exist for simple sequences. The uniqueness of spirals (up to reflection) follows immediately by induction on the number of faces.

Definition 9. A strong spiral sequence is a simple sequence $s=\left(s_{1}, \ldots, s_{l}\right)$, so that

- $l=1$ or

- $l=2$ and $\operatorname{ex}(s)>-6$ or

- $l \geq 3$ and there is a spiral $S_{s}$ with the following properties:

(i) The boundary of $S_{s}$ is formed in cyclic order by faces $\operatorname{sp}(i), \operatorname{sp}(i+$ $1), \ldots, \operatorname{sp}(l)$ for some $1 \leq i<l$.

(ii) For $j>i$ face $\operatorname{sp}(j)$ has a path with at least 2 edges in the boundary.

(iii) Face $\operatorname{sp}(l)$ has a path with at least 3 edges in the boundary. 
Lemma 10. Let $s=\left(s_{1}, \ldots, s_{l}\right)$ be a strong spiral sequence and $s^{\prime}=$ $\left(s_{1}, \ldots, s_{l}, s_{l+1}, \ldots s_{l^{\prime}}\right)$ a simple sequence that has $s$ as a prefix.

If $s_{l+1} \geq 6$, then $s^{\prime}$ is a strong spiral sequence too.

Proof. We will prove the lemma by induction and only have to describe the step $l \rightarrow l+1$ for each $l$. The case $l=1$ is trivial and for $l=2$, faces $\operatorname{sp}(1), \operatorname{sp}(2), \operatorname{sp}(3)$ form the boundary after extending the sequence with an entry $s_{3} \geq 6$. Face $\operatorname{sp}(1)$ has at least 2 edges in the boundary of $S_{\left(s_{1}, s_{2}\right)}$, so after adding a new face, at least 1 edge remains. Face $\operatorname{sp}(2)$ has at least 3 edges in the boundary of $S_{\left(s_{1}, s_{2}\right)}$, so after adding a new face at least 2 edges remain. Face $\operatorname{sp}(3)$ has at least 4 edges in the boundary of $S_{\left(s_{1}, s_{2}, s_{3}\right)}$, so $\left(s_{1}, s_{2}, s_{3}\right)$ is a strong spiral sequence too.

We start with a spiral for $s=\left(s_{1}, \ldots, s_{l}\right)$ with properties (i), (ii), and (iii). assuming that the faces in the boundary of this spiral are $\operatorname{sp}\left(l_{0}\right), \ldots, \operatorname{sp}(l)$, we just have to observe that face $\operatorname{sp}(l+1)$ must contain the common boundary vertex of $\operatorname{sp}\left(l_{0}\right)$ and $\operatorname{sp}(l)$ together with one edge of $\operatorname{sp}(l)$ and one edge of $\operatorname{sp}\left(l_{0}\right)$. If $\operatorname{sp}\left(l_{0}\right)$ had only one edge in the boundary, the new cyclic order is $\operatorname{sp}\left(l_{0}+1\right), \ldots, \operatorname{sp}(l+1)$, as $\operatorname{sp}\left(l_{0}+1\right)$ had at least two edges in the boundary before. The face $\operatorname{sp}(l+1)$ shares one edge with $\operatorname{sp}(l)$, one with $\operatorname{sp}\left(l_{0}\right)$ and at most one with $\operatorname{sp}\left(l_{0}+1\right)-\operatorname{sosp}(l+1)$, which has at least 6 edges, has at least 3 edges in the boundary and $\operatorname{sp}\left(l_{0}+1\right)$ at least one. If $\operatorname{sp}\left(l_{0}\right)$ had more than one edge in the boundary, the new cyclic order is $\operatorname{sp}\left(l_{0}\right), \ldots, \operatorname{sp}(l+1)$ and (ii) and (iii) follow analogously.

For some simple sequences $s=\left(s_{1}, \ldots, s_{l}\right)$ the boundary length does not monotonically increase when building the spiral one face at a time. This causes problems in some inequalities when comparing the bounday length with the boundary length of smaller c-6-patches. The following lemmas shed some light on the structure of these problematic sequences.

Lemma 11. All simple sequences $s=\left(s_{1}, \ldots, s_{l}\right)$ with $c(s)<6$ are strong spiral sequences and all simple sequences $s$ with $c(s)=6$ are either strong spiral sequences or the prefix $s^{\prime}$ of $s$ consisting of the $k$ elements smaller than 6 has a spiral with even boundary length $p$ and the degrees of the vertices in the boundary alternate between 2 and 3 . In this case we call $s$ a weak spiral sequence and $s^{\prime}$ a weak prefix.

If $s=\left(s_{1}, \ldots, s_{l}\right)$ is a weak spiral sequence with weak prefix $\left(s_{1}, \ldots, s_{k}\right)$ and $s_{j}>6$ for some $j>k$, then $l<\min \left\{m \mid m>j\right.$ and $\left.p-k \equiv 0\left(\bmod \frac{p}{2}\right)\right\}$, so the sequence $s$ contains less than $\frac{p}{2}$ elements larger than 6 . All bounded faces with more than 6 edges are in the boundary of $S_{s}$ and have at most 4 internal edges. 


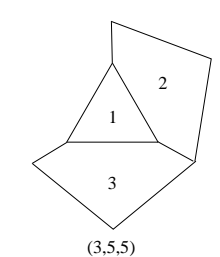

$(3,5,5)$
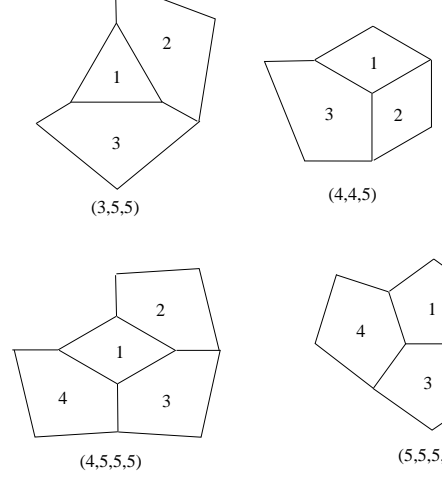

$(4,4,5)$

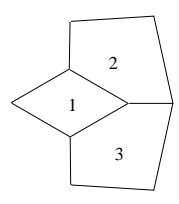

$(4,5,5)$
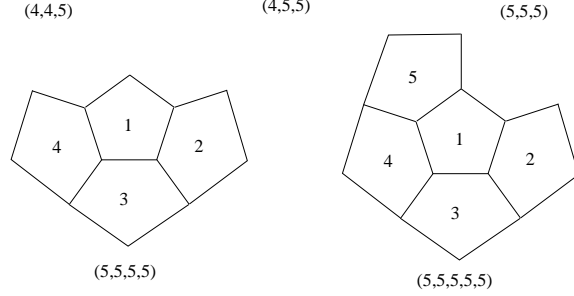

Figure 1: Spirals with at least 3 bounded faces, curvature less than 6 and all bounded faces of size at most 5 .

Proof. Except in the case $s_{1}=s_{2}=3$ where the vertices in the boundary of the spiral have degrees $2,3,2,3$ in this order, for $l \leq 2$ or $s_{3} \geq 6$ already the prefix $s=\left(s_{1}, s_{2}\right)$ is a strong spiral sequence and therefore due to Lemma 10 also $s$. The spirals for all other possible prefixes consisting of the elements that are smaller than 6 are given in Figure 1 and Figure 2. Checking these cases proves the first part of the lemma.

For the second part it is sufficient to note that for $m=k+x \cdot \frac{p}{2}$ for some $x>0$ and $s^{\prime \prime}=\left(s_{1}, \ldots, s_{k}, s_{k+1}^{\prime \prime}, \ldots, s_{m}^{\prime \prime}\right)$ a simple sequence with prefix $\left(s_{1}, \ldots, s_{k}\right)$, the spiral $S_{s^{\prime \prime}}$ has a boundary of length $p$ and the degrees of the vertices in the boundary alternate between 2 and 3 if $s_{m}^{\prime \prime}=6$ and that $s^{\prime \prime}$ is a strong spiral sequence if $s_{m}^{\prime \prime}>6$.

Lemma 12. Let $s=\left(s_{1}, \ldots, s_{l}\right)$ be a weak simple sequence with weak prefix $s^{\prime}=\left(s_{1}, \ldots, s_{k}\right)$. For $k \leq i \leq l$ we have that

$$
b\left(S_{s, i}\right)-\operatorname{ex}\left(S_{s, i}\right) \in\left\{b\left(S_{s^{\prime}}\right)-\operatorname{ex}\left(s^{\prime}\right), b\left(S_{s^{\prime}}\right)-\operatorname{ex}\left(s^{\prime}\right)+2\right\} .
$$

Let $p=\frac{b\left(S_{s^{\prime}}\right)}{2}$. If $i+p \leq l$ then $b\left(S_{s, i}\right)-\operatorname{ex}\left(S_{s, i}\right)=b\left(S_{s, i+p}\right)-\operatorname{ex}\left(S_{s, i+p}\right)$.

Proof. As for the spiral of the weak prefix the degrees of the vertices in the boundary alternate between 2 and 3, we can easily check that by adding hexagons, $b(\cdot)-\operatorname{ex}(\cdot)$ first grows by 2 - in fact $b(\cdot)$ grows and $\operatorname{ex}(\cdot)$ stays constant - until $p$ hexagons have been added. At that point $b(\cdot)$ is decreased by 2 and we are back at the same boundary structure and length. This proves the periodic behaviour of $b(\cdot)-\operatorname{ex}(\cdot)$ if only hexagons are added. If during 

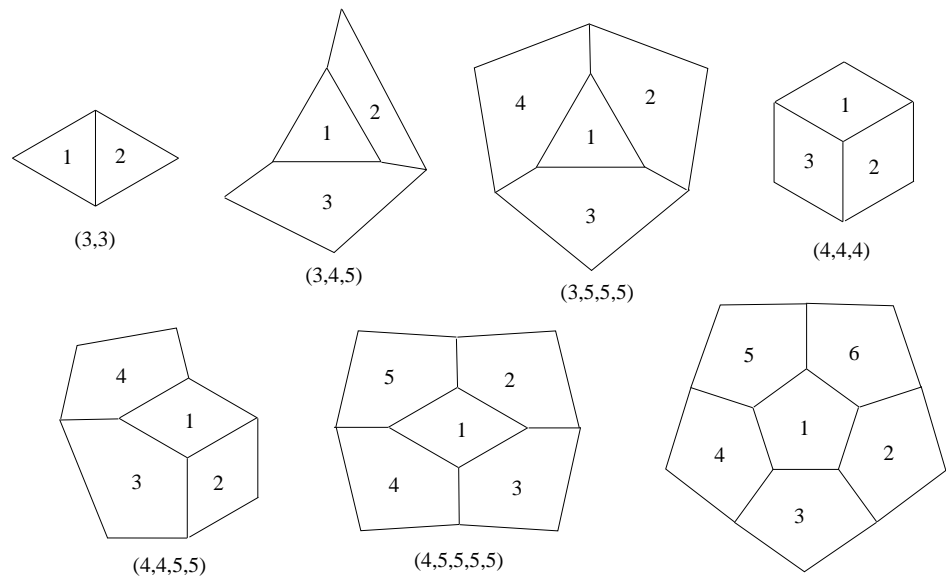

$(5,5,5,5,5,5)$

Figure 2: Spirals with curvature 6 and all bounded faces of size at most 5 .

one of the additions an entry $s_{i}>6$ occurs, the values of $b(\cdot)$ and $\operatorname{ex}(\cdot)$ are both increased by an additional $s_{i}-6$, so $b(\cdot)-\operatorname{ex}(\cdot)$ is the same as in the case of $s_{i}=6$.

Note that if $s_{l}>6$, and $i$ is the maximal index so that for $S_{s, i}$ the degrees of the vertices in the boundary alternate between 2 and 3 , then $l-i<p$, as for $l \geq i+p$ the sequence $s$ would either be a strong spiral sequence (if $s_{i+p}>6$ ) or for $S_{s, i+p}$ the degrees of the vertices in the boundary would alternate between 2 and 3 - contradicting the maximality of $i$.

In Figure 2 all spirals with curvature 6 and all face sizes smaller than 6 are displayed. From this figure we see that the only weak prefixes are $(3,3)$, $(3,5,5,5),(4,4,4),(4,5,5,5,5)$, and $(5,5,5,5,5,5)$.

Definition 13. Let $S_{s}$ be a spiral for a simple sequence $s=\left(s_{1}, \ldots, s_{l}\right)$ and $s^{+}=\max \left\{6, s_{l}\right\}$. A spiral $S_{\mathrm{cl}(s)}$ is called an enclosing spiral for $S_{s}$ if $\operatorname{cl}(s)=\left(s_{1}, \ldots, s_{l}, s_{l+1}, \ldots, s_{m}\right)$ with $s_{l+1}=s_{l+2}=\cdots=s_{m}=s^{+}$, $\operatorname{sp}(1), \ldots, \operatorname{sp}(l)$ are interior faces and $m$ is minimal with this property.

Lemma 14. Let $s=\left(s_{1}, \ldots, s_{l}\right)$ be a strong spiral sequence and $l \geq 2$. Then $S_{s}$ has the following properties:

(i) There exists an enclosing spiral $S_{\mathrm{cl}(s)}$.

(ii) Each face in the boundary of $S_{\mathrm{cl}(s)}$ shares an edge with exactly two boundary faces. 
(iii) If $s^{\prime}=\left(s_{1}, \ldots, s_{l}, s_{l+1}, \ldots, s_{l^{\prime}}\right)$ is a simple sequence with $s$ as prefix and with $s_{l+1} \geq 6$ then $b\left(S_{s}\right)-\operatorname{ex}(s) \leq b\left(S_{s^{\prime}}\right)-\operatorname{ex}\left(s^{\prime}\right)$. A special case is $b\left(S_{s}\right)-\operatorname{ex}(s) \leq b\left(S_{\mathrm{cl}(s)}\right)-\operatorname{ex}(\operatorname{cl}(s))$.

(iv) The interior faces of $S_{\mathrm{cl}(s)}$ are exactly $\operatorname{sp}(1), \ldots, \operatorname{sp}(l)$.

Proof. Item (i) follows immediately from the fact that a spiral for $s^{\prime}=$ $\left(s_{1}, \ldots, s_{l}, s_{l+1}, \ldots, s_{m}\right)$ for arbitrarily large $m$ exists (Lemma 10) and from the fact that the last face contains an edge of the smallest numbered face in the boundary.

Due to Definition 8, Lemma 10 and Definition 9 each boundary face of $S_{\mathrm{cl}(s)}$ shares edges with at least two other boundary faces. We will argue in the inner dual $D$ of $S_{\mathrm{cl}(s)}$. Assume now that there are vertices with degree 3 or more in the subgraph $G$ of $D$ induced by the vertices of $D$ corresponding to boundary faces. Then $G$ contains a subgraph consisting of the boundary cycle and a chord that can be described as the union of two cycles $C_{1}$, $C_{2}$ sharing an edge and with disjoint interior. In $D$, one of them, say $C_{1}$, contains all vertices corresponding to faces $\operatorname{sp}(1), \ldots, \operatorname{sp}(l)$ in the interior. Let $i$ be an index, so that $\operatorname{sp}(i) \in C_{2}$, but $\operatorname{sp}(i) \notin C_{1}$. This implies that $\operatorname{sp}(i)$ has no edge in common with $\operatorname{sp}(1), \ldots, \operatorname{sp}(l)$, so $S_{\mathrm{cl}(s), i-1}$ has none of the faces $\operatorname{sp}(1), \ldots, \operatorname{sp}(l)$ in the boundary - contradicting the minimality of the enclosing spiral. This implies (ii).

Item (iii) follows from item (ii) of Definition 9] and Lemma [10 which imply that for $i>l$ each face $\operatorname{sp}(i)$ shares at most 3 edges with $S_{s^{\prime}, i-1}$. This again implies that $b\left(S_{s^{\prime}, i}\right) \geq b\left(S_{s^{\prime}, i-1}\right)-3+\left(s_{i}-3\right)$. On the other hand $\operatorname{ex}\left(s_{1}, \ldots, s_{i-1}\right)+\left(s_{i}-6\right)=\operatorname{ex}\left(s_{1}, \ldots, s_{i}\right)$. Combining this we get

$$
\begin{aligned}
& b\left(S_{s^{\prime}, i}\right)-\operatorname{ex}\left(s_{1}, \ldots, s_{i}\right) \geq\left(b\left(S_{s^{\prime}, i-1}\right)+\left(s_{i}-6\right)\right)-\left(\operatorname{ex}\left(s_{1}, \ldots, s_{i-1}\right)+\left(s_{i}-6\right)\right) \\
& \quad=b\left(S_{s^{\prime}, i-1}\right)-\operatorname{ex}\left(s_{1}, \ldots, s_{i-1}\right) .
\end{aligned}
$$

Item (iv) is a consequence of items (i) and (ii) of Definition 9 and of Lemma 10. If $S_{\mathrm{cl}(s)}$ has $m$ bounded faces, then $\operatorname{sp}(m)$ shares an edge with $\operatorname{sp}(l)$ which is a boundary face in the spiral $S_{-}$without $\operatorname{sp}(m)$. As $\operatorname{sp}(l+1)$ has at least two edges in the boundary of $S_{-}$it has at least one edge in the boundary of $S_{\mathrm{cl}(s)}$, so $\operatorname{sp}(l+1), \ldots, \operatorname{sp}(m)$ are no interior faces.

Definition 15. For a simple sequence $s=\left(s_{1}, \ldots, s_{l}\right)$ and $1 \leq j \leq l$ we write $\operatorname{ex}(s, j)=\sum_{i=1}^{j}\left(s_{i}-6\right)$.

We say that a simple sequence $s=\left(s_{1}, \ldots, s_{l}\right)$ is dominated by a simple sequence $s^{\prime}=\left(s_{1}^{\prime}, \ldots, s_{l}^{\prime}\right)$ (of the same length) and write $s \preceq s^{\prime}$ if for all $1 \leq j \leq l$ we have that $\operatorname{ex}(s, j) \leq \operatorname{ex}\left(s^{\prime}, j\right)$. 
Theorem 16. Let $s, s^{\prime}$ be two simple sequences such that $s \preceq s^{\prime}$. Then for the spiral $S_{s}$ and every $s^{\prime}$-patch $P$ we have that

$b\left(S_{s}\right)-\operatorname{ex}\left(S_{s}\right) \leq b(P)-\operatorname{ex}(P)$.

As $s \preceq s$ this implies $b\left(S_{s}\right)=\min (s)$.

Proof. Assume that for some $m \geq 6$ there are sequences $s=\left(s_{1}, \ldots, s_{l}\right)$ and $s^{\prime}=\left(s_{1}^{\prime}, \ldots, s_{l}^{\prime}\right)$, so that the spiral $S_{s}$ and the $s^{\prime}$-patch $P$ are counterexamples (that is: $s \preceq s^{\prime}$ and $b\left(S_{s}\right)-\operatorname{ex}\left(S_{s}\right)>b(P)-\operatorname{ex}(P)$ ) with $s_{l} \leq m$ and $s_{l}^{\prime} \leq m$.

Among all these sequences choose $s, s^{\prime}$ so that the string $o\left(s^{\prime}, s\right)=$ $\left[-l, s_{1}^{\prime}, s_{1}, s_{2}^{\prime}, s_{2}, \ldots, s_{l}^{\prime}, s_{l}\right]$ is lexicographically maximal. Note that this implies that the number of faces is minimal. All bounded faces in the boundary of $P$ have size $m$, as otherwise we could subdivide boundary edges to obtain faces of size $m$ without changing $b(P)-\operatorname{ex}(P)$, but resulting in a sequence $s^{\prime}$ with larger $o\left(s^{\prime}, s\right)$ while still $s \preceq s^{\prime}$.

By Lemmas 3 and 5 we have

$b(P)-\operatorname{ex}(P)=2 v_{3, b}(P)+6 \geq 2 f_{b}(P)+6$ and

$b\left(S_{s}\right)-\operatorname{ex}\left(S_{s}\right)=2 v_{3, b}\left(S_{s}\right)+6=2 f_{b}\left(S_{s}\right)+6$

as boundary faces in spirals have a connected intersection with the boundary.

This implies that $f_{b}(P)<f_{b}\left(S_{s}\right)$ and especially $f_{b}(P)<l$, so $P$ has an interior face.

Removing the $b$ boundary faces of $P$, Lemma 7 gives for the resulting patchset $P_{-}$that $b\left(P_{-}\right)-\operatorname{ex}\left(P_{-}\right) \leq b(P)-\operatorname{ex}(P)-2\left(6+\operatorname{ex}\left(P_{-}\right)\right)$and with $P_{0}$ the patch with minimal boundary length for the resulting face sequence $s_{-}^{\prime}$ we have by Lemma 4 that $b\left(P_{0}\right)-\operatorname{ex}\left(P_{0}\right) \leq b(P)-\operatorname{ex}(P)-2\left(6+\operatorname{ex}\left(P_{-}\right)\right)$. As only faces of size $m$ were removed, we have $s_{-}^{\prime}=\left(s_{1}^{\prime}, \ldots, s_{l-b}^{\prime}\right)$, so for $s_{-}=\left(s_{1}, \ldots, s_{l-b}\right)$ we have $s_{-} \preceq s_{-}^{\prime}$.

We have that $s_{l-b+1}=\cdots=s_{l}=m$, as otherwise we could replace these boundary faces by $m$-gons and the modified sequence $s_{\text {mod }}$ would fulfill $b\left(S_{s_{\text {mod }}}\right)-\operatorname{ex}\left(S_{s_{\text {mod }}}\right)=b\left(S_{s}\right)-\operatorname{ex}\left(S_{s}\right)$, but as $s_{-} \preceq s_{-}^{\prime}$ and $s_{l-b+1}^{\prime}=\cdots=$ $s^{\prime}{ }_{l}=m$, we would have $s_{\text {mod }} \preceq s^{\prime}$ and $s_{\text {mod }}, s^{\prime}$ would be a counterexample with larger $o\left(s^{\prime}, s_{\text {mod }}\right)$.

We will distinguish between a strong and a weak spiral sequence $s$.

- Assume first that $s$ is a strong spiral sequence.

As $S_{s_{-}}$has more faces than $S_{s}$ has interior faces, we have that the enclosing spiral $S_{\mathrm{cl}\left(s_{-}\right)}$has more faces than $S_{s}$ and by Lemma 14 (iii) $b\left(S_{s}\right)-\operatorname{ex}(s) \leq b\left(S_{\mathrm{cl}\left(s_{-}\right)}\right)-\operatorname{ex}\left(\operatorname{cl}\left(s_{-}\right)\right)$. Removing the boundary faces of $S_{\mathrm{cl}\left(s_{-}\right)}$again we get $S_{s_{-}}$(Lemma 14(iv)) and due to Lemma14 (ii) we can apply Lemma 7 with equality and get

$$
b\left(S_{s_{-}}\right)-\operatorname{ex}\left(S_{s_{-}}\right)=b\left(S_{\mathrm{cl}\left(s_{-}\right)}\right)-\operatorname{ex}\left(\operatorname{cl}\left(s_{-}\right)\right)-2\left(6+\operatorname{ex}\left(s_{-}\right)\right) \geq
$$




$$
\begin{aligned}
& b\left(S_{s}\right)-\operatorname{ex}(s)-2\left(6+\operatorname{ex}\left(s_{-}\right)\right) \geq b\left(S_{s}\right)-\operatorname{ex}(s)-2\left(6+\operatorname{ex}\left(s_{-}^{\prime}\right)\right)> \\
& b(P)-\operatorname{ex}(P)-2\left(6+\operatorname{ex}\left(P_{-}\right)\right) \geq b\left(P_{0}\right)-\operatorname{ex}\left(P_{0}\right) .
\end{aligned}
$$

This contradicts the maximality of $o\left(s^{\prime}, s\right)$.

- Assume now that $s$ is a weak spiral sequence with weak prefix $s_{w}$. As in $s_{-}$only faces of size at least 6 were removed, $s_{w}$ is also a weak prefix of $s_{-}$.

By Lemma 12 we have

$b\left(S_{s_{-}}\right)-\operatorname{ex}\left(s_{-}\right) \in\left\{b\left(S_{s_{w}}\right)-\operatorname{ex}\left(s_{w}\right), b\left(S_{s_{w}}\right)-\operatorname{ex}\left(s_{w}\right)+2\right\}$

and $b\left(S_{s}\right)-\operatorname{ex}(s) \in\left\{b\left(S_{s_{w}}\right)-\operatorname{ex}\left(s_{w}\right), b\left(S_{s_{w}}\right)-\operatorname{ex}\left(s_{w}\right)+2\right\}$.

As the pair $P, S_{s}$ is a counterexample and due to Lemma 6 we have $b(P)-\operatorname{ex}(P) \leq b\left(S_{s_{w}}\right)-\operatorname{ex}\left(s_{w}\right)$. Due to the maximality of $o\left(s^{\prime}, s\right)$ we have that $P_{0}, S_{s_{-}}$form no counterexample, so $b\left(P_{0}\right)-\operatorname{ex}\left(P_{0}\right) \geq$ $b\left(S_{s_{w}}\right)-\operatorname{ex}\left(s_{w}\right)$ and together with Lemma 7 we get

$$
b\left(P_{0}\right)-\operatorname{ex}\left(P_{0}\right)=b\left(P_{-}\right)-\operatorname{ex}\left(P_{-}\right)=b(P)-\operatorname{ex}(P)=b\left(S_{s_{w}}\right)-\operatorname{ex}\left(s_{w}\right) .
$$

Again according to Lemma 7 this implies that $\operatorname{ex}\left(P_{-}\right)=-6$ and that each face in the boundary of $P$ has exactly two boundary faces as neighbours, which implies that the intersection of each boundary face with the boundary is connected. Now Lemmas 5$]$ and 3 imply

$$
f_{b}(P)=\frac{b(P)-6-\operatorname{ex}(P)}{2}=\frac{b\left(P_{-}\right)-6-\operatorname{ex}\left(P_{-}\right)}{2}=\frac{b\left(S_{s_{w}}\right)}{2} .
$$

As $P_{0}, S_{s_{-}}$form no counterexample, we have also $b\left(S_{s_{-}}\right)-\operatorname{ex}\left(s_{-}\right)=$ $b\left(S_{s_{w}}\right)-\operatorname{ex}\left(s_{w}\right)$. Due to Lemma12 this implies $b\left(S_{s}\right)-\operatorname{ex}(s)=b\left(S_{s_{-}}\right)-$ $\operatorname{ex}\left(s_{-}\right)=b\left(S_{s_{w}}\right)-\operatorname{ex}\left(s_{w}\right)$, in contradiction to the pair $P, S_{s}$ being a counterexample.

Theorem 16 now allows to compute the minimum boundary length for a given set of bounded faces. If one fixes the maximum face size to a value $m \geq 6$ and the boundary length is a strictly monotonically growing function of the number of faces, the theorem also solves the reverse question:

Given a certain value $b$ for the boundary length and a certain simple sequence $s$ with face sizes smaller than $m$. How many faces with size $m$ can a patch with boundary length $b$ and a face sequence with the given prefix $s$ and otherwise only bounded faces of size $m$ have? 
Lemma 17 gives formulas for the minimal boundary length for maximum face size 6 of a bounded face and at least one triangle or quadrangle as presented in [8] and corrected in [11]. The formulas for minimum face size 5 are already given in [2] and for minimum face size 6 in [10].

Lemma 17. [8][11] In the following equations $h$ stands for the number of elements with value 6 in the sequence.

- $\min ((4,6, \ldots, 6))=2\lceil\sqrt{8 h+4}\rceil$

- $\min ((4,5,6, \ldots, 6))=2\left\lceil\sqrt{6 h+\frac{49}{4}}+\frac{1}{2}\right\rceil-1$

- $\min ((4,5,5,6, \ldots, 6))=2\lceil\sqrt{4 h+16)}\rceil$

- $\min ((4,5,5,5,6, \ldots, 6))=2\left\lceil\sqrt{2 h+\frac{73}{4}}+\frac{1}{2}\right\rceil-1$

- if $h \equiv 0(\bmod 4)$ then $\min ((4,5,5,5,5,6, \ldots, 6))=8$ else $\min ((4,5,5,5,5,6, \ldots, 6))=10$

- $\min ((4,4,6, \ldots, 6))=2\lceil\sqrt{4 h+9)}\rceil$

- $\min ((4,4,5,6, \ldots, 6))=2\left\lceil\sqrt{2 h+\frac{49}{4}}+\frac{1}{2}\right\rceil-1$

- $\min ((4,4,5,5,6, \ldots, 6))=8$

- if $h \equiv 0(\bmod 3)$ then $\min ((4,4,4,6, \ldots, 6))=6$ else $\min ((4,4,4,6, \ldots, 6))=8$

- $\min ((3,6, \ldots, 6))=2\left\lceil\sqrt{6 h+\frac{9}{4}}+\frac{1}{2}\right\rceil-1$

- $\min ((3,5,6, \ldots, 6))=2\lceil\sqrt{4 h+8)}\rceil$

- $\min ((3,5,5,6, \ldots, 6))=2\left\lceil\sqrt{2 h+\frac{41}{4}}+\frac{1}{2}\right\rceil-1$

- if $h \equiv 0(\bmod 3)$ then $\min ((3,5,5,5,6, \ldots, 6))=6$ else $\min ((3,5,5,5,6, \ldots, 6))=8$

- $\min ((3,4,6, \ldots, 6))=2\left\lceil\sqrt{2 h+\frac{25}{4}}+\frac{1}{2}\right\rceil-1$

- $\min ((3,4,5,6, \ldots, 6))=6$

- if $h \equiv 0(\bmod 2)$ then $\min ((3,3,6, \ldots, 6))=4$ else $\min ((3,3,6, \ldots, 6))=6$

For the proofs we refer the reader to [11]. 


\section{Boundary lengths between the maximum and minimum}

We determined the minimum and maximum boundary length that can be achieved for a given simple sequence $s=\left(s_{1}, \ldots, s_{l}\right)$. As due to Lemma 6 $b(P)-\operatorname{ex}(P)$ is always even, the parity of the boundary length and the sum of face sizes must be the same and one can only hope for intermediate boundary lengths in steps of size two.

Definition 18. A simple sequence $s=\left(s_{1}, \ldots, s_{l}\right)$ is called diamond-free, if $l<2$ or $s_{2} \neq 3$.

Lemma 19. Two bounded faces in a c-6-patch $P$ without two neighbouring triangles share at most one edge.

Proof. Assume that a counterexample exists. Let $P$ be a counterexample with the smallest number of faces, and $f, f^{\prime}$ two bounded faces sharing more than one edge. It is easy to see that no two of these edges share a vertex as (using the fact that boundaries of faces in 2-connected graphs are cycles) this would be an internal vertex with degree 2 .

If we connect the centers $c_{f}$ and $c_{f^{\prime}}$ of $f$ resp. $f^{\prime}$ with the centers of all $k \geq 2$ common edges, we get $k$ disjoint paths from $c_{f}$ to $c_{f^{\prime}}$ partitioning the plane into $k$ components, only one of which contains the unbounded face. Let $J$ be the Jordan curve bounding a component without the outer face.

The interior of $J$ is not empty and contains only bounded faces (different from $f, f^{\prime}$ ). The union of these faces is a patch $P^{\prime}$ bounded by a cycle consisting of a part from the boundary of $f$ and a part from the boundary of $f^{\prime}$. As all vertices of $P^{\prime}$ have degree 3 in $P$, all except two vertices - the endpoints of the edges intersected by $J$ - have degree 3 in $P^{\prime}$. So for $P^{\prime}$ we have $v_{2, b}=2$ and by Lemma 3 we have $v_{3, b} \leq 2$. In case $v_{3, b}<2$ there would be a double edge or a bridge, so $v_{3, b}=2$. If the two vertices with degree 3 in the boundary are not connected by an edge through the interior of $P^{\prime}$, the patch $P^{\prime}$ contradicts the minimality of $P$ - otherwise $P^{\prime}$ consists of two neighbouring triangles - in contradiction to the assumption.

Lemma 20. Let $l$ be given and $m=\max \{6, l\}$. If for each diamond-free simple sequence $s=\left(s_{1}, \ldots, s_{l}\right)$ with $s_{l} \leq m$ and for all even $0 \leq c \leq$ $\max (s, 1)-\min (s)$, an s-patch $P$ with $b(P)=\min (s)+c$ exists, then this also holds without the restriction $s_{l} \leq m$.

Proof. For a diamond-free sequence $s=\left(s_{1}, \ldots, s_{l}\right)$ let $s_{r}=\left(s_{r, 1}, \ldots, s_{r, l}\right)$ denote the reduced sequence where all elements larger than $m$ are replaced by $m$. As $m \geq 6$ we have that $c(s)=c\left(s_{r}\right)$ and $s_{r}$ is also a simple sequence. 
We will show that if for the reduced sequence all intermediate boundary lengths with the necessary parity exist, then also for the original sequence.

As for given $s$ the value of $\operatorname{ex}(P)$ is constant for every $s$-patch $P$, we can equivalently discuss whether for all even $d$ with $\min (s)-\operatorname{ex}(s) \leq d \leq$ $\max (s, 1)-\operatorname{ex}(s)$ there is an $s$-patch $P$ with $b(P)-\operatorname{ex}(P)=d$.

Due to Lemma 19 each bounded face of size $q \geq l$ in an $s$-patch shares at most $l-1<q$ edges with other bounded faces, so all faces with size at least $l$ are in the boundary.

To this end we can modify a spiral for $s_{r}$ to a spiral for $s$ by just subdividing a boundary edge in those faces that correspond to values that were replaced. Such an operation does not change $b(\cdot)-\operatorname{ex}(\cdot)$, so we have $b\left(S_{s}\right)-\operatorname{ex}(s)=b\left(S_{s_{r}}\right)-\operatorname{ex}\left(s_{r}\right)$. The same operation can be done on all $s_{r}$-patches with boundary lengths up to $\max \left(s_{r}, 1\right)$ proving that if for all even $d$ with $\min \left(s_{r}\right)-\operatorname{ex}\left(s_{r}\right) \leq d \leq \max \left(s_{r}, 1\right)-\operatorname{ex}\left(s_{r}\right) s_{r}$-patches $P$ with $b(P)-\operatorname{ex}(P)=d$ exist, the corresponding result also holds for $s$.

The fact that we excluded patches with two triangles in these lemmas is not simply because the proof does not work. In fact the main result of this section - that is that for all values (with the suitable parity) between the minimum and maximum boundary length patches exist - is not true for patches with two triangles. E.g. for the sequence $s=(3,3,7,8)$ c-6patches with boundary length $b$ exist for each odd $b$ with $\min (s)=7 \leq$ $b \leq 15=\max (s, 1)$ - except for $b=9$. For $s=(3,3,6,6,6,6)$ c-6-patches with boundary length $b$ exist for each even $b$ with $\min (s)=4 \leq b \leq 20=$ $\max (s, 1)$ - except for $b=6$. For $s=(3,3,6,6,6,6,6)$ c-6-patches with boundary length $b$ exist for each even $b$ with $\min (s)=6 \leq b \leq 24=$ $\max (s, 1)$ - except for $b=10$. Though all missing values we know of seem to display a certain pattern, we will not investigate this very special case here.

Theorem 21. Let $s=\left(s_{1}, \ldots, s_{l}\right)$ be a simple diamond-free sequence. Then for all even $0 \leq c \leq \max (s, 1)-\min (s)$, an s-patch $P$ with $b(P)=\min (s)+c$ exists.

Proof. The cases $s=\left(s_{1}, \ldots, s_{l}\right)$ with $l \leq 4$ can be easily checked by hand, as due to Lemma 20 it is sufficient to check sequences with $s_{l} \leq 6$. So we can assume $l \geq 5$. One can also easily check all simple sequences with at most one entry larger than 5 (that is: $s_{l-1}<6$ and implies $l \leq 7$ ) by hand. Alternatively one can (as we in fact did) use the program described in [4] to generate all patches for these simple sequences and combine it with an easy program to check the boundary lengths. The generation program 
is publically available in the environment CaGe [3]. We applied it for all sequences $s=\left(s_{1}, \ldots, s_{l}\right)$ with $l \leq 9, s_{l} \leq 9$. All cases where intermediate boundary lengths were missing, were sequences with $s_{1}=s_{2}=3$.

We will prove that for all even $d$ with $\min (s)-\operatorname{ex}(s) \leq d \leq \max (s, 1)-$ $\operatorname{ex}(s)$ there is an $s$-patch $P$ with $b(P)-\operatorname{ex}(P)=d$.

As before, we call a boundary edge with both endpoints of degree 2 a convex edge and we call a boundary path of length 2 with the central vertex of degree 3 and the two endpoints of degree 2 a concave path.

We will first prove that for all diamond-free simple sequences $s^{\prime}=$ $\left(s_{1}^{\prime}, \ldots, s_{l}^{\prime}\right)$ with $l \geq 5$ and for all even $d \in\left\{\min \left(s^{\prime}\right)-\operatorname{ex}\left(s^{\prime}\right)+2, \min \left(s^{\prime}\right)-\right.$ $\left.\operatorname{ex}\left(s^{\prime}\right)+4\right\}$ there is an $s^{\prime}$-patch $P$ with $b(P)-\operatorname{ex}(P)=d$ and a convex boundary edge $e$.

The boundary length of a patch with face sequence $s=\left(s_{1}, \ldots, s_{l}\right)$ and $i$ interior edges is $\sum_{j=1}^{l} s_{j}-2 i$. So removing a face of a patch with boundary length $b$ and excess ex that has $j$ interior edges and gluing it back along $j^{\prime}$ edges results in a patch with $b^{\prime}-\mathrm{ex}^{\prime}=(b-\mathrm{ex})+2\left(j-j^{\prime}\right)$.

Assume that $s^{\prime}$ as well as $\left(s_{1}^{\prime}, \ldots, s_{l-1}^{\prime}\right)$ and $\left(s_{1}^{\prime}, \ldots, s_{l-2}^{\prime}\right)$ are strong spiral sequences. This is e.g. the case for $c\left(s^{\prime}\right)<6$ and implies that in the spirals for $s^{\prime}$ and $\left(s_{1}^{\prime}, \ldots, s_{l-1}^{\prime}\right)$ the last face has 2 or 3 interior edges, as in the case of 4 edges one would have two bounded faces with only one boundary edge for the spiral with one face less. Furthermore these spirals have a convex edge as well as a concave path. If the last face has 3 interior edges, one can remove it and put it back identifying an edge with a convex edge of the remaining patch (resulting in a patch with $\left.b^{\prime}-\mathrm{ex}^{\prime}=(b-\mathrm{ex})+4\right)$ or a path of length 2 with a concave path (resulting in a patch with $\left.b^{\prime}-\mathrm{ex}^{\prime}=(b-\mathrm{ex})+2\right)$. If the last face has 2 interior edges, by removing and gluing it back along a convex edge one gets a patch with $b^{\prime}-\mathrm{ex}^{\prime}=(b-\mathrm{ex})+2$. In order to get a patch with $b^{\prime}-\mathrm{ex}^{\prime}=(b-\mathrm{ex})+4$ one can remove the last two faces (losing 4 or 5 interior edges) and glue the first face back along a concave path or a convex edge and the second along a convex edge of the first face. As the faces removed and glued back are at least pentagons, they are guaranteed to have a convex edge after being glued back.

If one of $s^{\prime},\left(s_{1}^{\prime}, \ldots, s_{l-1}^{\prime}\right)$ and $\left(s_{1}^{\prime}, \ldots, s_{l-2}^{\prime}\right)$ is a weak spiral sequence, then assume first that $s_{l}^{\prime} \leq 6$. In that case $s^{\prime}$ itself is a weak spiral sequence and has a weak prefix $p^{\prime}$. With $b=b\left(S_{p^{\prime}}\right)$ we have $\min \left(s^{\prime}\right)-\operatorname{ex}\left(s^{\prime}\right) \in$ $\left\{b-\operatorname{ex}\left(s^{\prime}\right), b-\operatorname{ex}\left(s^{\prime}\right)+2\right\}$.

We will show that for $h \geq 2$ hexagons there are patches $P$ with $b(P)-$ $\operatorname{ex}(P)=d$ for all $d \in\left\{b-\operatorname{ex}\left(s^{\prime}\right)+2, b-\operatorname{ex}\left(s^{\prime}\right)+4, b-\operatorname{ex}\left(s^{\prime}\right)+6\right\}$ that have a convex edge as well as a concave path. Replacing $b-\operatorname{ex}\left(s^{\prime}\right)$ by the possible values for $\min \left(s^{\prime}\right)-\operatorname{ex}\left(s^{\prime}\right)$ this implies that for $h \geq 2$ hexagons there are 


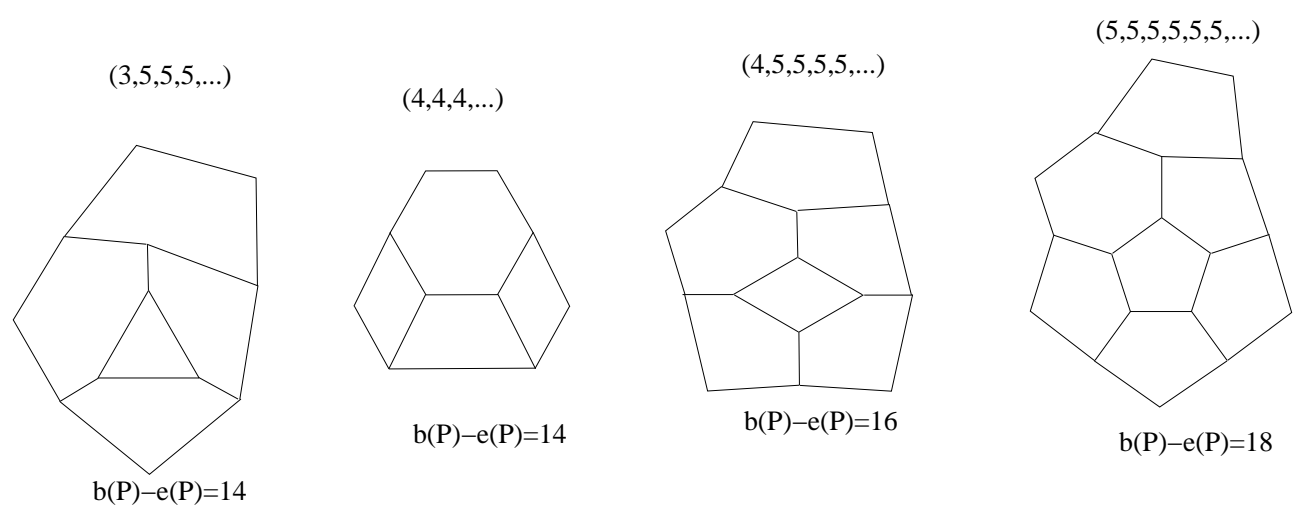

Figure 3: Patches with a boundary length $b\left(S_{p^{\prime}}\right)+2$ corresponding to weak prefixes $p^{\prime}$ plus one hexagon.

patches $P$ with $b(P)-\operatorname{ex}(P)=\min \left(s^{\prime}\right)-\operatorname{ex}\left(s^{\prime}\right)+2$ and patches $P$ with $b(P)-\operatorname{ex}(P)=\min \left(s^{\prime}\right)-\operatorname{ex}\left(s^{\prime}\right)+4$.

In Figure 3 patches for $b-\operatorname{ex}\left(s^{\prime}\right)+2$ are given for all weak prefixes and one hexagon. Successively adding hexagons at the place with two neighbouring boundary vertices of degree 3 we get for all $h \geq 1$ patches $P$ with the same value of $b(P)-\operatorname{ex}(P)$, a convex edge as well as a concave path and two neighbouring vertices of degree 3 in the boundary.

Adding a second hexagon along a concave path or a convex edge, we get patches with $b(\cdot)-\operatorname{ex}(\cdot)=b\left(S_{p^{\prime}}\right)+4$, resp. $b(\cdot)-\operatorname{ex}(\cdot)=b\left(S_{p^{\prime}}\right)+6$ for all $h \geq 2$. Note that for up to $b / 2$ hexagons all hexagons are in the boundary and that for more than $b / 2$ hexagons at least $b / 2$ hexagons are in the boundary of the patches we constructed.

If $s^{\prime}$ contains also faces of size larger than 6 , then the number is at most $b / 2$ (Lemma 11). As they are all in the boundary of the spiral $S_{s^{\prime}}$, we have $\min \left(s^{\prime}\right)-\operatorname{ex}\left(s^{\prime}\right)=\min \left(s^{\prime \prime}\right)-\operatorname{ex}\left(s^{\prime \prime}\right)$ with $s^{\prime \prime}$ the sequence where all elements larger than 6 are replaced by 6 . Furthermore we can modify boundary faces of the patches with $b(\cdot)-\operatorname{ex}(\cdot) \in\left\{b-\operatorname{ex}\left(s^{\prime}\right)+2, b-\operatorname{ex}\left(s^{\prime}\right)+4, b-\operatorname{ex}\left(s^{\prime}\right)+6\right\}$ constructed for $s^{\prime \prime}$ to obtain examples for $s^{\prime}$.

Now we will proceed by induction in $l$ and show that for all diamondfree simple sequences $s^{\prime}$ and for all even $d$ with $\max \left(s^{\prime}, 1\right)-\operatorname{ex}\left(s^{\prime}\right) \geq d>$ $\min \left(s^{\prime}\right)-\operatorname{ex}\left(s^{\prime}\right)+4$ there is an $s^{\prime}$-patch $P$ with $b(P)-\operatorname{ex}(P)=d$ and a convex boundary edge.

Let $s^{\prime}=\left(s_{1}^{\prime}, \ldots, s_{l}^{\prime}\right)$ be a simple diamond-free sequence with $l \geq 5$, $s_{l-1} \geq 6$ and let $d$ be even with 
- $d>\min \left(s^{\prime}\right)-\operatorname{ex}\left(s^{\prime}\right)+4$

- $d<\max \left(s^{\prime}, 1\right)-\operatorname{ex}\left(s^{\prime}\right)$

Let $s_{-}^{\prime}=\left(s_{1}^{\prime}, \ldots, s_{l-1}^{\prime}\right)$. Then

- $d-4 \geq \min \left(s^{\prime}\right)-\operatorname{ex}\left(s^{\prime}\right)+2 \geq \min \left(s_{-}^{\prime}\right)-\operatorname{ex}\left(s_{-}^{\prime}\right)$ (Lemma 12, Lemma 14 (iii))

- $d-4<\max \left(s_{-}^{\prime}, 1\right)-\operatorname{ex}\left(s_{-}^{\prime}\right)($ Lemma 2)

The first inequality is an equality only if $\operatorname{sp}(l)$ is the last face in a layer around a boundary with alternating degrees of 2 and 3. In that case the minimal spiral for $s_{-}^{\prime}$ has also a convex edge. So there exists an $s_{-}^{\prime}$-patch $P_{-}$with $b\left(P_{-}\right)-\operatorname{ex}\left(P_{-}\right)=d-4$ and a convex boundary edge. Identifying an edge of a face of size $s_{l}$ with this boundary edge, we get a patch $P$ with $b(P)-\operatorname{ex}(P)=\left(b\left(P_{-}\right)+s_{l}-2\right)-\left(\operatorname{ex}\left(P_{-}\right)+s_{l}-6\right)=d-4+4=d$.

\section{References}

[1] P. Bonsma and F. Breuer. Finding fullerene patches in polynomial time. In 20th International Symposium on Algorithms and Computations (ISAAC 2009), volume 5878 of Lecture Notes in Computer Science, 2009.

[2] J. Bornhöft, G. Brinkmann, and J. Greinus. Pentagon-hexagon-patches with short boundaries. European Journal of Combinatorics, 24:517-529, 2003.

[3] G. Brinkmann, O. Delgado-Friedrichs, S. Lisken, A. Peeters, and N. Van Cleemput. Cage - a virtual environment for studying some special classes of plane graphs - an update. MATCH Commun. Math. Comput. Chem., 63(3):533-552, 2010. http://caagt.ugent.be/CaGe/.

[4] G. Brinkmann and P. Goetschalckx. Enumeration of generalized fusenes. MATCH Commun. Math. Comput. Chem., 74(3):505-520, 2015.

[5] R.H. Buchholz and W. de Launey. An edge-minimization problem for regular polygons. Electronic Journal of Combinatorics, 16(1), 2009. Article Number: R90. 
[6] V.M. Buchstaber and N.Y. Erokhovets. Constructions of families of three-dimensional polytopes, characteristic patches of fullerenes, and Pogorelov polytopes. Izvestiya Mathematics, 81(5):901-972, 2017.

[7] S.J. Cyvin and J. Brunvoll. Series of benzenoid hydrocarbons with a constant number of isomers. Chem. Phys. Lett., 176(5):413-416, 1991.

[8] A. Egging. Kürzeste Randlängen von Patches mit Drei-, Vier- Fünfund Sechsecken. Master's thesis, Universität Bielefeld, 2005.

[9] L. Faria, S. Klein, and M. Stehlík. Odd cycle transversals and independent sets in fullerene graphs. SIAM Journal on Discrete Mathematics, 26(3):1458-1469, 2012.

[10] F. Harary and H. Harborth. Extremal animals. J. of Comb., Inf. \& Syst. Sci., 1:1-8, 1976.

[11] C. Justus. Boundaries of triangle-patches and the expander constant of fullerenes. PhD thesis, Universität Bielefeld, 2007.

[12] F. Kardoš. A computer-assisted proof of Barnette-Goodey conjecture: Not only fullerene graphs are hamiltonian. https://arxiv.org/abs/1409.2440.

[13] D. Nicodemos and M. Stehlík. Packing and covering odd cycles in cubic plane graphs with small faces. European Journal of Combinatorics, 67:208-221, 2018. 